\title{
Elastic-net regularization approaches for genome-wide association studies of rheumatoid arthritis

\author{
Seoae Cho ${ }^{1}$, Haseong $\mathrm{Kim}^{2}$, Sohee $\mathrm{Oh}^{3}$, Kyunga $\mathrm{Kim}^{3}$ and Taesung Park*1,3
}

Addresses: ${ }^{1}$ Interdisciplinary Program in Bioinformatics, Seoul National University, Kwan-ak St. 599, Kwan-ak Gu, Seoul, South Korea 151-741, Republic of Korea, ${ }^{2}$ Department of Electrical and Electronic Engineering, Imperial College London, SW7 2AZ, UK and ${ }^{3}$ Department of Statistics, Seoul National University, Kwan-ak St. 599, Kwan-ak Gu, Seoul, South Korea 151-741, Republic of Korea

E-mail: Seoae Cho - sacho71@snu.ac.kr; Haseong Kim - kimhaseong@gmail.com; Sohee Oh - oh.sohee@gmail.com;

Kyunga Kim - kyunga.j.kim@gmail.com; Taesung Park* - tspark@stats.snu.ac.kr

${ }^{*}$ Corresponding author

from Genetic Analysis Workshop 16

St Louis, MO, USA 17-20 September 2009

Published: 15 December 2009

BMC Proceedings 2009, 3(Suppl 7):S25 doi: 10.1186/I753-656I-3-S7-S25

This article is available from: http://www.biomedcentral.com/I753-656I/3/S7/S25

(c) 2009 Cho et al; licensee BioMed Central Ltd.

This is an open access article distributed under the terms of the Creative Commons Attribution License (http://creativecommons.org/licenses/by/2.0), which permits unrestricted use, distribution, and reproduction in any medium, provided the original work is properly cited.

\begin{abstract}
The current trend in genome-wide association studies is to identify regions where the true diseasecausing genes may lie by evaluating thousands of single-nucleotide polymorphisms (SNPs) across the whole genome. However, many challenges exist in detecting disease-causing genes among the thousands of SNPs. Examples include multicollinearity and multiple testing issues, especially when a large number of correlated SNPs are simultaneously tested. Multicollinearity can often occur when predictor variables in a multiple regression model are highly correlated, and can cause imprecise estimation of association. In this study, we propose a simple stepwise procedure that identifies disease-causing SNPs simultaneously by employing elastic-net regularization, a variable selection method that allows one to address multicollinearity. At Step I, the single-marker association analysis was conducted to screen SNPs. At Step 2, the multiple-marker association was scanned based on the elastic-net regularization. The proposed approach was applied to the rheumatoid arthritis (RA) case-control data set of Genetic Analysis Workshop 16. While the selected SNPs at the screening step are located mostly on chromosome 6, the elastic-net approach identified putative RA-related SNPs on other chromosomes in an increased proportion. For some of those putative RA-related SNPs, we identified the interactions with sex, a well known factor affecting RA susceptibility.
\end{abstract}

\section{Background}

Recently, genome-wide association studies (GWAS) have become a promising new tool for deciphering the genetics of complex diseases, which are usually polygenic and affected by gene-by-environmental interactions. Because it can be more powerful to scan multiple markers jointly in detecting disease-related genes, various multiplemarker approaches have been or can be used in GWAS [1-4]. Examples include logic regressions [2] and classification and regression trees [3]. Due to their 
sequential selection processes, these methods may miss the overall correlation structure of the genes. Another example is random forest [4], based on which true disease-causing genes can be hidden due to other genes; the identification result may not be robust.

In this study, we propose a simple stepwise procedure that employs the elastic-net regularization-based approach [5] to take the overall correlation structure of single-nucleotide polymorphisms (SNPs) into account when selecting disease-causing genes automatically in GWAS. Because the elastic net imposes on a combination of lasso and ridge penalties $[6,7]$, it provides a more reproducible prediction than using multiple regression, especially when there are highly correlated predictors (e.g., SNPs in high linkage disequilibrium). Our approach consists of two main steps, called the screen step and the elastic-net step. At the screen step, we eliminate most of noise SNPs via single-marker association tests, and select the largest number of candidate SNPs that can be analyzed by the elastic-net approach at the next step. At the elastic-net step, putative disease-causing SNPs are jointly identified based on multiple logistic regressions with the screened SNPs via the elastic net. Interactions between SNP and non-genotypic factor (e.g., sex) can also be examined.

The proposed approach was applied to the rheumatoid arthritis (RA) case-control dataset of Genetic Analysis Workshop 16 (GAW16). RA is a complex disease with a moderately strong genetic component. It is generally known that females are at a higher risk than males and the mean onset of disease is in the fifth decade. Many studies have implicated the HLA region on chromosome 6 p21, with consistent evidence for several DR alleles contributing to risk [8]. Among the non-HLA loci, PTPN22 on chromosome 1p13, a gene coding for protein tyrosine non-receptor22, is considered as a strong candidate RA-susceptibility gene [9]. Recently, a functional SNP in this PTPN22 gene was reported to be associated with RA [10]. There remains much to learn about the genetic susceptibility for RA, including possible gene-by-environmental interactions.

\section{Methods}

\section{Genotype data and sample}

The RA data from GAW16 included 545,080 SNPs genotyped by Illumina (550 k chip) along with covariates for 908 cases and 1260 controls. We adjusted population stratification using the computer program Eigenstrat [11] by excluding 20 outliers from the samples. Also, the samples showing sex matching error were filtered [12]. We excluded SNPs with $>10 \%$ missing genotype, with minor allele frequencies $<5 \%$, and/or with $p<0.001$ from Hardy-Weinberg equilibrium tests. As a result, 474,499 SNPs passed our quality control filters and were used in the proposed stepwise analyses.

\section{Step I: Screening SNPs via single-marker association tests} For each single SNP, the disease association is tested using the following logistic regression model adjusted by sex, under the additive mode of inheritance:

$$
\log \operatorname{it}(\pi)=\beta_{0}+\beta_{1} S E X+\beta_{2} S N P_{i}
$$

where $\pi$ represents the probability of getting the disease. Among the SNPs showing the strongest associations, we select the largest number of SNPs that can be analyzed in the penalized logistic regression via the elastic net at the next step. This screening step is needed to address the computational limitation when applying the penalized logistic regression via the elastic net to multiple SNPs.

\section{Step 2: Penalized logistic regression models via the elastic net}

In this step, putative disease-causing SNPs are identified via elastic-net-based variable selection. The elastic-net method is particularly useful when the number of highly correlated predictor variables $(p)$ is much larger than the sample size $(N)$. The elastic-net regularization approach solves the following problem:

$$
\min _{\left(\beta_{0}, \beta\right) \in \mathfrak{R}^{p+1}}\left[\frac{1}{2 N} \sum_{i=1}^{N}\left(\gamma_{i}-\beta_{0}-x_{i}^{T} \beta\right)^{2}+\lambda P_{\alpha}(\beta)\right],
$$

where the elastic-net penalty is defined as

$$
P_{\alpha}(\beta)=(1-\alpha) \frac{1}{2}\|\beta\|_{l_{2}}^{2}+\alpha\|\beta\|_{l_{1}}=\sum_{j=1}^{p}\left[\frac{1}{2}(1-\alpha) \beta_{j}^{2}+\alpha\left|\beta_{j}\right|\right] .
$$

The elastic-net penalty creates a useful a compromise between the ridge-regression penalty $(\alpha=0)$ [9] and the lasso penalty $(\alpha=1)$ [10]. The elastic net with $\alpha=1-\varepsilon$ for some small $\varepsilon>0$ performs much like the lasso, but is robust to extreme correlations among predictor variables. Moreover, the elastic net does both shrinkage and automatic variable selection simultaneously. The choice of the regularization parameter $(\lambda)$ is critical to selecting important variables with accurate estimation. Tuning parameters $\alpha$ and $\lambda$ are usually selected to minimize mean-squared prediction error based on cross-validations (e.g., 5-fold).

Because the effect of genotype variations (i.e., SNPs) on disease status can be modified by other factors (in our study, sex), we consider the following multiple logistic 
regression models to examine the SNP main effects (M1) and also interaction effects of SNPs with sex (M2).

$$
\begin{gathered}
\operatorname{logit}(\pi)=\beta_{0}+\beta_{1} S E X+\sum_{i=1}^{N_{S N P}} \beta_{2 i} S N P_{i} \\
\operatorname{logit}(\pi)=\beta_{0}+\beta_{1} S E X+\sum_{i=1}^{N_{S N P}} \beta_{2 i} S N P_{i}+\sum_{i=1}^{N_{S N P}} \beta_{12 i} S E X * S N P_{i},
\end{gathered}
$$

where $\pi$ represents the probability of getting the disease. When M1 is used with the elastic-net penalties, the SEX variable is not penalized to adjust the sex effect in selecting SNP main effects. Note that main effect terms of both SEX and SNPs are not penalized when examining the SNP-by-sex interactions in M2. In this study, we use a library 'glmnet' in $\mathrm{R}$ statistical package http://www. r-project.org to conduct the penalized logistic regressions via the elastic-net.

\section{Results}

\section{Single-marker associations}

The single-marker association test was conducted for each SNP, and 48,336 SNPs showed $p$-values below 0.05 (Figure 1). Some SNPs are in HLA-DRB1 and PTPN22, which were already known to be RA-susceptibility genes [8-10]. Among the 48,336 SNPs, we chose the top 1000, 2000, and 3000 significant SNPs for Step 2.

\section{Main effect analysis via elastic-net (MI)}

We applied the model M1 via the elastic net to top 1000, 2000, and 3000 SNPs selected at the first step. Among top 1000 SNPs, 250 SNPs were identified with main effects as putative RA-related SNPs while 360 SNPs were detected among the top 2000 and 398 SNPs among the top 3000. Those with the ten largest main effects are listed in Table 1. The resulting putative RA-related SNPs are displayed across the whole genome in Figure 1. Across the screening choices, 81 SNPs were commonly selected. Among those SNPs, 23 SNPs were identified also from single-marker association analyses after 5\% Bonferroni multiplicity correction, and (except three SNPs) are located on chromosome 6. For examples, rs2395175 and rs660895 in HLA-DRB1 and HLA-DRA on chromosome 6 had $p$-values of $1.08 \times 10^{-87}$ and $7.16 \times$ $10^{-90}$, respectively, from single-marker association test. However, 58 overlapping SNPs that were not identified from single-marker association analyses were found on various chromosomes. Some SNPs are located on known genes, such as AMFR, ANKRD35, ECT2, TARBP1, ZFP92, and ZFPM2. For instance, rs2440468 is located in AMFR (autocrine motility factor receptor) gene on chromosome 16. AMF secretion and receptor levels are closely related to RA as well as tumor malignancy [13]. Note that RA-susceptibility odds ratios (ORs) of AG and GG against AA were 0.78 and 0.57 , respectively, for this SNP. However, rs 2440468 had a $p$-value $=5.74 \times 10^{-5}$ for singlemarker association test. While the evidence for singlemarker based association at chromosome 6 with RA has been previously identified by numerous studies [1], our

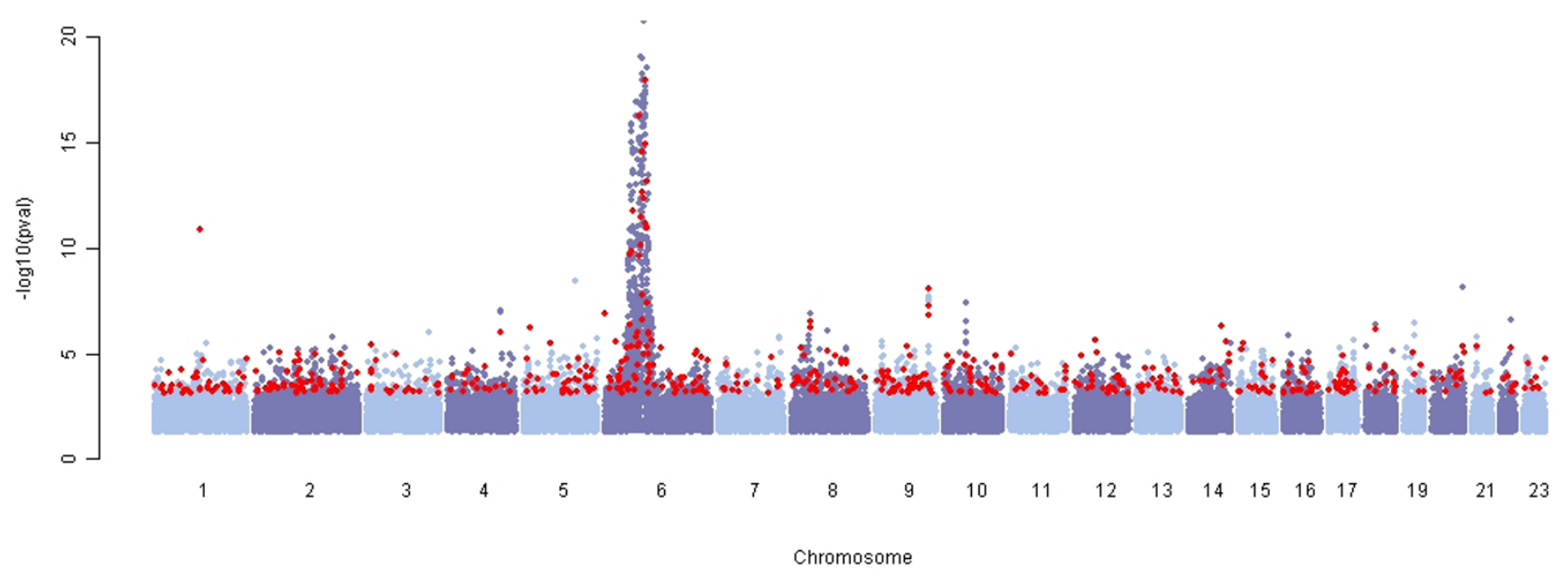

Figure I

Genome-wide scan for RA-SNP association. The $p$-values $<0.05$ from single SNP association tests were plotted in - $\log _{10}$ scale against chromosomal positions of the corresponding 48,366 SNPs. Blue and light blue were used to distinguish chromosomes. Red indicates potential RA-related SNPs that were identified by fitting the penalized logistic regression model (MI) via elastic-net using top 3000 of those 48,366 SNPs. 
Table I: RA-related SNPs identified with ten largest main effects via the elastic-net method (MI)

\begin{tabular}{|c|c|c|}
\hline SNP & Chromosome $^{a}$ & Coefficient ${ }^{\mathrm{b}}$ \\
\hline \multicolumn{3}{|l|}{ Top 1000} \\
\hline rs6903608 & 6 & -0.3413 \\
\hline rs2395I85 & 6 & 0.3285 \\
\hline rsll 686264 & 2 & -0.3284 \\
\hline rs698|223 & 8 & -0.31 \\
\hline rsl0948693 & 6 & -0.2813 \\
\hline rs9727917 & 1 & -0.2806 \\
\hline rs2440468 & 16 & -0.2736 \\
\hline rs4499874 & 5 & $0.27 \mid 4$ \\
\hline rs9275595 & 6 & 0.2641 \\
\hline rs7970893 & 12 & -0.2492 \\
\hline \multicolumn{3}{|l|}{ Top 2000} \\
\hline rs2395।75 & 6 & 0.2522 \\
\hline rs6903608 & 6 & -0.2299 \\
\hline rs 10094729 & 8 & -0.166 \\
\hline$r s 2101613$ & 10 & -0.1613 \\
\hline rs691007I & 6 & 0.1529 \\
\hline rs660895 & 6 & 0.1522 \\
\hline rs9277554 & 6 & -0.1468 \\
\hline rs 12203592 & 6 & -0.1401 \\
\hline rs 2578240 & 9 & 0.1356 \\
\hline rs9275572 & 6 & -0.1353 \\
\hline \multicolumn{3}{|l|}{ Top 3000} \\
\hline rs2395175 & 6 & 0.3532 \\
\hline rs660895 & 6 & 0.2302 \\
\hline rs9275572 & 6 & -0.219 \\
\hline rs 10094729 & 8 & -0.1972 \\
\hline rs 6903608 & 6 & -0.1889 \\
\hline rs3873444 & 6 & -0.1403 \\
\hline rs7970893 & 12 & -0.1321 \\
\hline rs234592 & 14 & -0.1316 \\
\hline rs10789176 & 1 & -0.125 \\
\hline rs927560I & 6 & -0.1221 \\
\hline
\end{tabular}

${ }^{a}$ Chromosome where SNP is located.

${ }^{\mathrm{b}}$ Coefficient representing size and direction of SNP main effect.

results indicate that putative RA-related SNPs were also distributed across several other regions outside of the chromosome 6 (Figure 2).

\section{Interaction analysis with sex via elastic-net (M2)}

To investigate SNPs with effects on RA-susceptibility that varied across sexes, we performed interaction analysis (M2) with the putative RA-related SNPs from M1 for each screening choice (i.e., top 1000, 2000, and 3000). We identified 71 SNPs and 132 SNPs with the SNP-bysex interaction for each choice of top 1000 and top 2000, while 105 SNPs showed interactions for top 3000 choice. Those with five largest interactions effects are summarized in Table 2. For each sex, we investigated RAsusceptibility OR of each genotype against major-allele homozygote. For example, rs2044750 showed heterozygote OR of 1.12 and 1.71 for female and male, respectively. The OR for AA is 1.37 for female and 2.37 for male. This SNP is located in nuclear factor of activated T cell 1 (NFATc1), a transcription factor on chromosome 18, which has recently been shown to be related to osteoporosis, bone metastasis, and rheumatoid arthritis [14]. Note that rs2044750 showed a nonsmall $p$-value of 0.00041 at single-marker association test. Note that ten SNPs overlapped across the screening choices. Out of ten SNPs, we found six SNPs in known genes, such as C19orf2, CUGBP2, ECT2, TBC1D8, and WNT3.

\section{Discussion}

We have proposed a simple stepwise approach that employs the multiple logistic regression model with the elastic-net penalties to detect disease-causing genes across a whole genome in GWAS. The elastic-net method using both lasso and ridge penalties has several advantages in identifying disease-causing SNPs jointly in GWAS. First, automatic variable selection and continuous shrinkage can be simultaneously performed. Second, it can select groups of many highly correlated SNPs, which may cause a multicollinearity problem in classical multiple linear regressions. Third, the shrinkage feature of the elastic net enables us to include all the interaction terms between SNPs and non-genotypic factors as well as SNP main effects into a model. Also, rather than searching for potential SNPs along the entire chromosome directly, our approach provides an efficient search by using a multi-step procedure to handle the extremely large number of potential SNP patterns in GWAS.

Although most putative RA-related SNPs were found in chromosome 6, we also identified additional susceptibility genes in other chromosomes. Our findings need to be replicated in an independent dataset or to be functionally validated in the future in order to declare the biological significance. There is disagreement in results across the screening choices. There are possible causes that result in this discrepancy. First, the missing data caused large differences in the results. We removed some samples and SNPs to make datasets complete because the elastic-net regression method we employed does not allow for missing data. So the three datasets according to the screening choices ended up with different sample sizes. The difference in sample size was large in the previous analysis, and we tried to make the sample sizes similar in the updated analysis. Even though the previous analysis had a similar sample size, there are about only $70 \%$ overlapping samples, as shown below. This explains why we had more common SNPs in the updated results. This missing data problem would be avoided by using a proper imputation method for missing data. Second, depending on the correlation structures among SNPs, the elastic-net regression method may provide different results because 


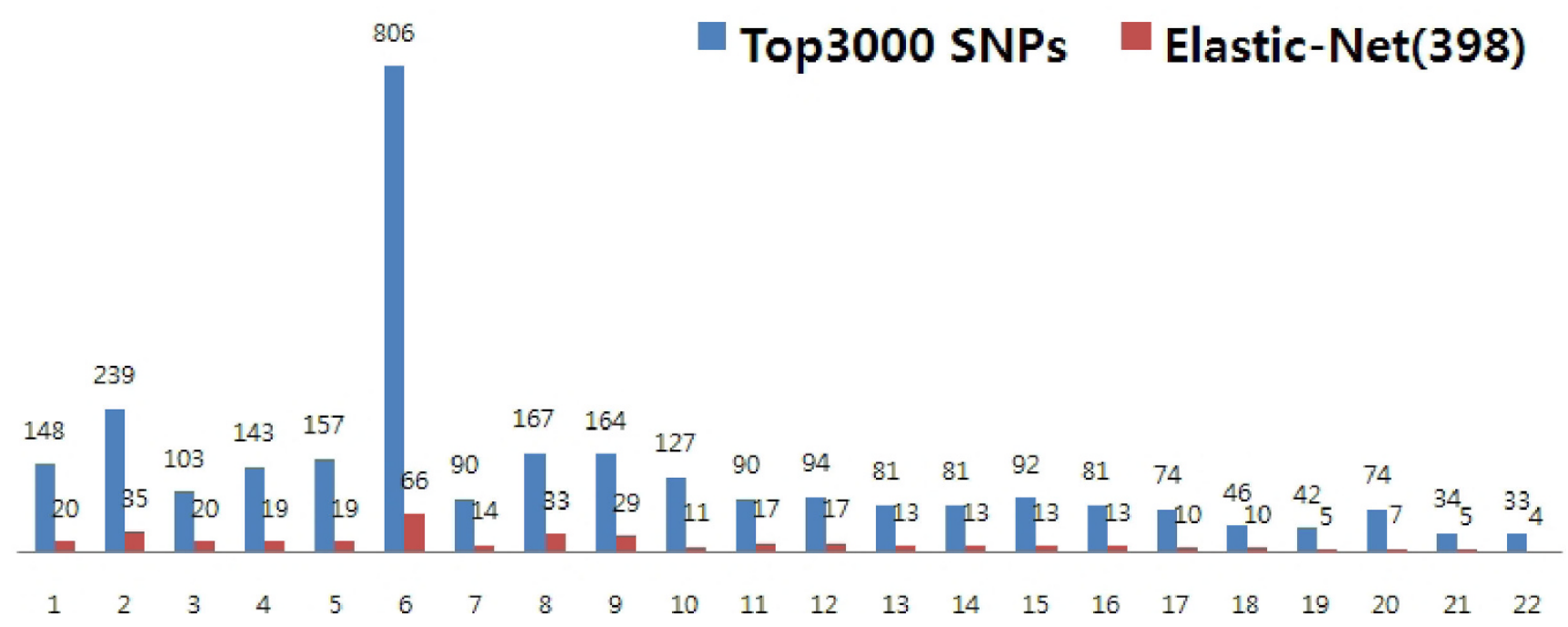

Figure 2

Distributions of top 3000 screened SNPs vs. 398 potential RA-related SNPs across chromosomes. For each chromosome, blue bars represent the number of SNPs that were selected as top 3000 SNPs via single SNP association tests at Step I; and red bars represent the number of potential RA-related SNPs that were identified at Step 2 by fitting penalized logistic regression model (MI) via elastic-net using the top 3000 screened SNPs.

Table 2: RA-related SNPs identified with sex-by-SNP interaction via the elastic-net method (M2)

\begin{tabular}{lcc}
\hline SNP & Chromosome $^{\mathbf{a}}$ & Coefficient $^{\mathbf{b}}$ \\
\hline Top 1000 & & \\
rs2858870 & 6 & -0.329 \\
rs9727917 & 1 & -0.2572 \\
rs10184573 & 2 & -0.2347 \\
rs105149II & 17 & 0.2314 \\
rs11703I5I & 22 & 0.2077 \\
Top 2000 & & \\
rs6903608 & 6 & -0.538 \\
rs1217675 & 8 & 0.5188 \\
rs56027I & 17 & -0.5169 \\
rs201119 & 10 & -0.4943 \\
rs2044750 & 18 & -0.4812 \\
Top 3000 & & \\
rs3873444 & 6 & -0.3573 \\
rs948195 & 11 & -0.3233 \\
rs2579088 & 12 & 0.3063 \\
rs13277II3 & 8 & 0.303 \\
rs12407970 & 1 & 0.2787 \\
\hline
\end{tabular}

${ }^{a}$ Chromosome where SNP is located.

${ }^{\mathrm{b}}$ Coefficient representing size and direction of SNP main effect.

it considers the correlation structure when selecting variables.

\section{List of abbreviations used}

GAW16: Genetic Analysis Workshop 16; GWAS: Genome-wide association study; OR: Odds ratio; RA: Rheumatoid arthritis; SNPs: Single-nucleotide polymorphisms.

\section{Competing interests}

The authors declare that they have no competing interests.

\section{Authors' contributions \\ HK and SO participated in statistical analysis. SC participated in the design of the study, performed the statistical analysis, and drafted the manuscript. KK and TP conceived of the study, and participated in its design and coordination and helped to draft the manuscript. All authors read and approved the final manuscript.}

\section{Acknowledgements}

The Genetic Analysis Workshops are supported by NIH grant ROI GM03 I575 from the National Institute of General Medical Sciences. The work was supported by the National Research Laboratory Program of Korea Science and Engineering Foundation (MI0500000 I26).

This article has been published as part of BMC Proceedings Volume 3 Supplement 7, 2009: Genetic Analysis Workshop 16. The full contents of the supplement are available online at http://www.biomedcentral.com/ |753-656|/3 ? issue=S7.

\section{References}

I. Ye $\mathrm{Y}$, Zhong $\mathrm{X}$ and Zhang $\mathrm{H}$ : A genome-wide tree- and forestbased association analysis of comorbidity of alcoholism and smoking. BMC Genetics 2005, 6(SuppI I):SI 35

2. Schwendeer $\mathrm{H}$ and Ickstadt $\mathrm{K}$ : Identification of SNP interactions using logic regression. Biostatistics 2008, 9: 187-198.

3. Breiman L, Friedman J. Olshen $\mathrm{R}$ and Stone C: Classification and Regression Trees.New York: Chapman \& Hall; 1984

4. Breiman L: Random forests. Mach Learn 200I, 45:5-32.

5. Zou $\mathrm{H}$ and Hastie $\mathrm{T}$ : Regularization and variable selection via the elastic net. J Roy Stat Soc Ser B 2005, 67:30I-320. 
6. Tibshirani RJ: Regression shrinkage and selection via the LASSO. Roy Stat Soc Ser B 1996, 58:267-288.

7. Le Cessie $S$ and van Houwelingen JC: Ridge estimators in logistic regression. Appl Statist 1992, 41:|9|-20|.

8. Jawaheer D, Li W, Graham RR, Chen W, Damle A, Xiao X Monteiro J, Khalili $H$, Lee A, Lundsten R, Begovich A, Bugawan T, Erlich H, Elder JT, Criswell LA, Seldin MF, Amos Cl, Behrens TW and Gregersen PK: Dissecting the genetic complexity of the association between human leukocyte antigens and rheumatoid arthritis. Am J Hum Genet 2002, 71:585-594.

9. Begovich $A B$, Carlton VE, Honigberg LA, Schrodi SJ, Chokkalingam AP, Alexander HC, Ardlie KG, Huang Q, Smith AM, Spoerke JM, Conn MT, Chang M, Chang SY, Saiki RK, Catanese J], Leong DU, Garcia VE, McAllister LB, Jeffery DA, Lee AT, Batliwalla F, Remmers E, Criswell LA, Seldin MF, Kastner DL, Amos Cl, Sninsky JJ and Gregersen PK: A missense single-nucleotide polymorphism in a gene encoding a protein tyrosine phosphatase (PTPN22) is associated with rheumatoid arthritis. Am J Hum Genet 2004, 75:330-337.

10. Carlton VE, Hu X, Chokkalingam AP, Schrodi SJ, Brandon R, Alexander HC, Chang M, Catanese J], Leong DU, Ardlie KG, Kastner DL, Seldin MF, Criswell LA, Gregersen PK, Beasley E, Thomson G, Amos $\mathrm{Cl}$ and Begovich AB: PTPN22 genetic variation: evidence for multiple variants associated with rheumatoid arthritis. Am J Hum Genet 2005, 77:567-58I.

II. Price AL, Patterson NJ, Plenge RM, Weinblatt ME, Shadick NA and Reich D: Principal components analysis corrects for stratification in genome-wide association studies. Nat Genet 2006, 38:904-909.

12. Purcell S, Neale B, Todd-Brown K, Thomas L, Ferreira MA, Bender D, Maller J, Sklar P, de Bakker PI, Daly MJ and Sham PC PLINK: a tool set for whole-genome association and population-based linkage analyses. Am J Hum Genet 2007, 81:559-575.

13. Haga A, Funasaka T, Niinaka $Y$, Raz A and Nagase H: Autocrine motility factor signaling induces tumor apoptotic resistance by regulations Apaf-I and Caspase-9 apoptosome expression. Int J Cancer 2003, 107:707-7|4.

14. Tsuji-Naito K: Aldehydic components of cinnamon bark extract suppresses RANKL-induced osteoclastogenesis through NFATcl downregulation. Bioorg Med Chem 2008, 16:9176-9183.
Publish with Bio Med Central and every scientist can read your work free of charge

"BioMed Central will be the most significant development for disseminating the results of biomedical research in our lifetime. "

Sir Paul Nurse, Cancer Research UK

Your research papers will be:

- available free of charge to the entire biomedical community

- peer reviewed and published immediately upon acceptance

- cited in PubMed and archived on PubMed Central

- yours - you keep the copyright
BioMedcentral 